\title{
Clomipramine Administered during the Luteal Phase Reduces the Symptoms of Premenstrual Syndrome: A Placebo-Controlled Trial
}

Charlotta Sundblad, M.D., Marina A. Hedberg, M.D., and Elias Eriksson, M.D., Ph.D.

ha previous controlled trial we have shown that memenstrual irritability and depressed mood (vemenstrual syndrome) can be effectively reduced by boses of the potent (but nonselective) serotonin mptake inhibitor clomipramine taken each day of the mstrual cycle. The present study was undertaken to omine to what extent intermittent administration of domipramine, during the luteal phase only, is also fatioe against premenstrual complaints. Twenty-nine ondpressed women displaying severe premenstrual initetility and/or depressed mood and fulfilling the DSM-III-R criteria of late luteal phase dysphoric disorder ure treated daily from the day of ovulation until the of of the menstruation either with clomipramine (25 b $75 \mathrm{mg})(\mathrm{n}=15)$ or with placebo $(\mathrm{n}=14)$ for three macutive menstrual cycles; another nine subjects (seven a domipramine, two on placebo) dropped out during vatment. In both treatment groups self-rated monstrual irritability and depressed mood (as registered daily using a visual analogue scale) were significantly reduced during treatment; in the placebo group, this symptom reduction was about $45 \%$, whereas in the clomipramine group it was greater than $70 \%$. The mean premenstrual ratings of irritability and depressed mood during the three treatment cycles were significantly lower in the clomipramine group than in the placebo group. Also with respect to the rating of global improvement, the result obtained with clomipramine was significantly better than that obtained with placebo. The study confirms the previously reported effectiveness of low doses of clomipramine in the treatment of premenstrual syndrome and demonstrates that the time lag between onset of medication and clinical effect is shorter when clomipramine is used for premenstrual syndrome than when it is used for depression, panic disorder, or obsessive compulsive disorder.

[Neuropsychopharmacology 9:133-145, 1993] iv wORDS: Premenstrual syndrome; Late luteal phase Sphoric disorder; Clomipramine; Antidepressant; Sotonin reuptake inhibitor

from the Department of Pharmacology (CS, EE), and the Departof Psychiatry and Neurochemistry (MAH), University of GöteSweden

Address correspondence to: Elias Eriksson, M.D., Ph.D., Departof Pharmacology, University of Göteborg, Medicinaregatan 7, S113 90 Göteborg, Sweden.

Recived December 10, 1992; revised April 27, 1993; accepted May IIm.
The cardinal symptoms of the premenstrual syndrome (PMS), or late luteal phase dysphoric disorder (LLPDD), are irritability and depressed mood; in addition, tension, increased carbohydrate craving, sleep disturbance, and a sense of bloating are common complaints. For fulfillment of the diagnosis, the symptoms should start regularly around ovulation or during the two weeks preceding the menstrual bleeding and terminate within a few days after the onset of menstruation (Frank 1931; Halbreich et al. 1982; American Psychiatric Association 1985).

Most women of fertile age experience mild premen- 
strual complaints (Reid 1985; Andersch et al. 1986; Chihal 1987); needless to say, a majority of these women are capable of mastering their symptoms without medication. However, several independent studies indicate that as much as $10 \%$ of all menstruating women have premenstrual complaints of such severity that they would like to medicate to reduce them (Reid 1985; Andersch et al. 1986; Chihal 1987).

In a recent open study (Eriksson et al. 1990), followed by a confirmative placebo-controlled trial (Sundblad et al. 1992), we have shown that daily intake throughout the menstrual cycle of low doses of the antidepressant clomipramine very effectively reduces premenstrual sadness and irritability in nondepressed women suffering from LLPDD. It was hypothesized that the impressive effect of clomipramine in reducing premenstrual complaints was mainly due to the serotonin reuptake inhibiting effect of the drug.

In spite of the comparatively low doses used in these studies ( 25 to $75 \mathrm{mg}$ per day), side effects of the treatment were not uncommon. For this reason, several of the participating women expressed the opinion that intermittent administration of the drug during the premenstrual phase only would be more attractive than a medication given also during the symptom-free follicular phase. As judged by the clinical profile of clomipramine when used for the treatment of depression, panic disorder (Modigh et al. 1992), and obsessivecompulsive disorder (OCD) (Katz et al. 1990), one would not expect such a noncontinuous medication to be effective; thus, in most trials, clomipramine, like other monoamine reuptake inhibitors, requires at least 2 to 3 weeks of treatment to exert marked clinical effects. Hence, we were surprised when a small group of women with severe premenstrual complaints during an open pilot trial indeed reported a marked effect of clomipramine also when the drug was taken in the luteal phase only (Sundblad and Eriksson, unpublished finding). To investigate whether this response is indeed related to the pharmacodynamics of clomipramine or is merely a placebo effect, the present controlled trial of intermittent administration of clomipramine in severe LLPDD was undertaken.

\section{SUBJECTS AND METHODS}

\section{Recruitment and Inclusion Criteria}

Women with premenstrual irritability and/or depressed mood were recruited by means of a newspaper advertisement followed by a brief telephone interview and a subsequent, extensive, structured interview. The primary inclusion criteria were severe irritability and/or depressed mood starting regularly around ovulation or during the 2 weeks preceding the menstrual bleeding and terminating within a few days after the onset of menstruation as reported by the patient during the interview. In addition, the criteria of LLPDD in DSM-III-R (American Psychiatric Association 1985) should be fulfilled. Exclusion criteria were: 1) previous or ongoing psychiatric illness (apart from major depressive disor. der or dysthymic disorder), 2) ongoing major depres. sive disorder or dysthymic disorder (as defined using DSM-III-R and by means of the Montgomery-Äsherg Depression Scale), 3) major depressive disorder or dysthymic disorder less than 2 years from the time of the interview, 4) ongoing medication for somatic or ps!chiatric illness, 5) ongoing medication with oral contraceptives, 6) ongoing alcohol abuse, 7) ongoing somatic illness, 8) irregular menstruations, 9) ongoing or planned pregnancy, 10) less than 18 years of age, and 11) previous treatment with antidepressants for premenstrual complaints. Informed consent was obtained from all participants.

Before starting medication, all participants performed daily rating with respect to the possible occur. rence of irritability, depressed mood, tension, increased appetite and/or carbohydrate craving, breast tender. ness, and bloating, respectively, for two subsequent menstrual cycles using a visual analogue scale (VAS) ( 0 to $100 \mathrm{~mm} ; 0 \mathrm{~mm}=$ no complaints, $100 \mathrm{~mm}=$ maxi . mal complaints). Subjects not displaying the following evidence of menstrually related changes during both reference cycles were excluded from the study. 1) More than a $100 \%$ increase in either irritability or depressed mood (or both) during the premenstrual phase (calculated as the mean rating of the 5 days before the 1st day of menstruation) as compared to the postmenstrual phase (calculated as the mean rating of days 6 to $10 \mathrm{of}$ the cycle; cycle day 1 = the 1st day of menstruation). 2) Mean premenstrual rating of irritability or depressed mood exceeding $20 \mathrm{~mm}$. In addition, some participants were excluded during the course of the two reference cycles due to other reasons (such as pregnancy and onset of depression).

Immediately after the second reference cycle, the participants fulfilling the inclusion criteria were ran. domized either to the clomipramine or to the placebo group. For practical reasons a number of participants were included in the randomization before having comrpleted the rating of the second reference cycle; some of these turned out to display an unsatisfactory symp. tom rating during the reference cycles and were hence excluded in spite of the fact that they had been partol the randomization.

\section{Medication}

After the 2 months of symptom rating, medication started for patients not excluded due to unsatisfactor rating (or other reasons) during the reference cycles. Tablets containing placebo and clomipramine, resper. 
ively, were identical in shape, size, and color, and both pients and investigators were unaware of whether the patient was given placebo or clomipramine until the sudy was completed. Treatment started at the estiated time of ovulation (14 days before expected menoruation) and continued until the start of menstruaion. On the first day of medication, the patients were motructed to take one tablet containing $5 \mathrm{mg}$ of clomipromine (or placebo). The following day, the patients rok two tablets each containing $5 \mathrm{mg}$ of clomipramine mplacebo). The third day of medication, the patients rok one tablet containing $25 \mathrm{mg}$ clomipramine (or perebo). From the fourth day of treatment and onwards moughout the entire premenstrual period the patients wre instructed to take two tablets each containing 25 a of clomipramine (or placebo). However, patients operiencing marked side effects of the treatment were intructed to decrease the dose to one tablet daily. On ther hand, patients experiencing no or mild side fects and an unsatisfactory reduction of premenstrual complaints during the course of the first premenstrual phese were instructed to increase the dosage from two bthree tablets daily. Thus, the dosage applied was flexWe; two tablets (50 $\mathrm{mg}$ of clomipramine) being the reommended dose and one $(25 \mathrm{mg})$ and three $(75 \mathrm{mg})$ blets, respectively, being the minimal and maximal mes allowed. At the first day of menstruation, the paints were to reduce the dose to one tablet containing Jig (or placebo), the second day of menstruation the ptients took one tablet containing $10 \mathrm{mg}$ of clomipraine (or placebo), whereas the third day of menstruain the dose was $5 \mathrm{mg}$ of clomipramine (or one tablet d placebo). From the fourth day of menstruation until thestimated day of ovulation the patients were free bon medication. The same tablet regimen was repeated bring the two following treatment cycles.

\section{Serum Drug Concentrations}

Fordetermination of serum levels of clomipramine and ismetabolite desmethylclomipramine, blood samples me obtained in the morning 1 to 5 days before the montrual bleeding during treatment cycle 3 ; the preating intake of medication had occurred 8 PM the moning before. Serum levels of clomipramine and deswhyldomipramine were measured using gas chromawraphy as previously described (Nyberg and Mårusson 1984). The lower detection limit was $10 \mathrm{nM}$ for woh clomipramine and desmethylclomipramine.

\section{Eat Assessment}

Ouring the three treatment cycles the participants pertured daily symptom ratings using the same 1 to 100 n VAS (comprising the items irritability, depressed mod, tension, increased appetite/carbohydrate crav- ing, breast tenderness, and bloating) as during the two pretreatment reference cycles. In addition, symptoms were rated also during one additional drug-free reference cycle after the three cycles of medication were completed. To obtain a global assessment of possible drug effects, all patients were asked to estimate how they felt with respect to their premenstrual complaints during treatment as compared to how they felt before (enormously improved $=7$, much improved $=6$, somewhat improved $=5$, no change $=4$, somewhat deteriorated $=3$, much deteriorated $=2$, enormously deteriorated $=1$ ) within 2 weeks after the last treatment cycle. The spouses of all cohabiting participants were asked to perform an independent rating of the premenstrual condition of the patient with respect to irritability and depressed mood once every reference and treatment cycle using a 1 to $100 \mathrm{~mm}$ VAS. It was emphasized that this rating should be based on the spouse's own observations and that it should not be influenced by any preceding discussion between patient and cohabitor. Spouse's ratings were not mandatory.

\section{Tolerability Assessment}

Before the start of the trial, the participants were informed of the various side effects that could be expected from treatment with clomipramine and instructed to report, by telephone call or by a note in the symptom assessment form, any symptom appearing during medication for which there were no other obvious explanation. To what extent certain specified side effects were present was never actively inquired by the investigators.

\section{Statistics}

For statistical comparisons within and between the two treatment groups, the mean rating during the 5 days preceding the menstrualbleeding in each treatment cycle was used as a measure of premenstrual symptomatology, whereas the mean rating of cycle day 6 to 10 was used as a measure of postmenstrual complaints.

Within both treatment groups, the premenstrual symptom rating scores during the two reference cycles (mean of both cycles) were compared with the symptom rating during each of the three different treatment cycles as well as with the mean of all three treatment cycles using Wilcoxon signed rank test. In addition, the mean of the three treatment cycles was compared with the rating of the posttreatment reference cycle using the same statistical test.

With respect to the following items, the two treatment groups were compared with each other using the Mann-Whitney U test. 1) pre- and postmenstrual rating of the six symptoms inquired (irritability, depressed mood, tension, increased appetite/carbohydrate crav- 
ing, breast tenderness, bloating) during each reference cycle, during each treatment cycle, and during the posttreatment reference cycle, 2) the mean premenstrual symptom rating of the two pretreatment reference cycles and of the three treatment cycles (all six symptoms), respectively, 3) global improvement or deterioration, as rated by the patient shortly after the last treatment cycle, 4) spouse's rating of irritability and depressed mood before (mean of both reference cycle ratings) and during (mean of all available treatment cycle ratings) medication, 5) maximal dose of test drug or placebo.

In line with the protocol for the trial, only patients completing the study were included in the primary effect assessment. $p$ values less than .05 were regarded as statistically significant.

\section{RESULTS}

\section{Number of Drop Outs and Fulfillers}

Of 64 women recruited by means of a telephone interview and a structured interview (see Methods), nine women for varying reasons chose not to participate in the study. Another 17 subjects were excluded from the study for one or several of the following reasons. 1) They were found to fulfill one or more of the exclusion criteria during the two pretreatment reference cycles (see Methods). 2) They were found not to display a men- strually related cyclical pattern with respect to initabiity and/or depressed mood (as defined in Methods) dur. ing the two initial reference cycles.

Thirty-eight subjects started medication with eithe clomipramine $(n=22)$ or placebo $(n=16)$. The uneven distribution of subjects between the two treatment groups was accidental and due to the fact that randomi zation for some subjects was undertaken before they were excluded due to unsatisfactory rating during the two reference cycles.

Two placebo treated patients dropped out during the study; one because of side effects and one because of pregnancy. In the clomipramine group, seven pz tients dropped out; six because of side effects and one because of pregnancy. Consequently, 29 patients $(15$ on clomipramine and 14 on placebo) fulfilled the trial.

The mean age of the patients in the clomipramine group was $40.4 \pm 1.5$ years and in the placebo group $38.2 \pm 1.2$ years. Four of the patients completing the trial - two in each treatment group - reported that the had previously been treated with antidepressants fo depression. No other patients reported any previow episodes of affective illness.

\section{Medication}

After the dose titration undertaken during the first and to some extent, second treatment cycles, in the placebo

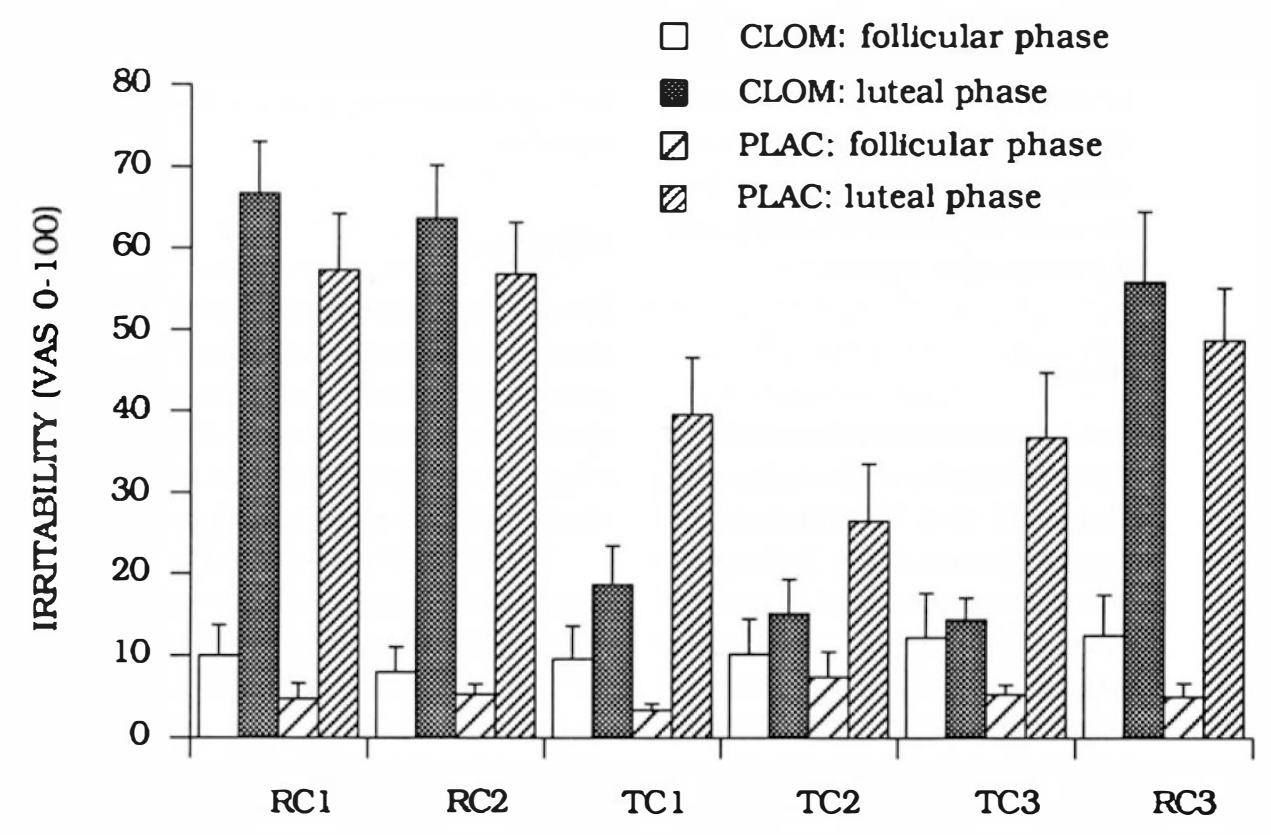

Figure 1. Means of self-rated irritability (VAS 0 to $100 \mathrm{~mm}$ ) of cycle day 6 to 10 (follicular phase) and of the five days precol ing the day of the menstruation (luteal phase). The bars represent group means with standard errors. During the two 00 secutive pretreatment reference cycles (RC1 and $R C 2)$ and during the posttreatment reference cycle (RC3), no treatmet was given, whereas in the treatment cycles (TC1 to TC3) the patients were medicated with clomipramine or placebo pre menstrually. Levels of significance (clomipramine group versus placebo group) for the premenstrual phases of each cyde were: RC1 NS, RC2 NS, TC1 $p=.04$, TC2 NS ( $p=.1)$, TC3 $p=.03$, RC3 NS. For levels of significance when comparing the ratings of the reference cycles with the rating of the treatment cycles within each treatment group, see Results. 
goup five subjects had chosen a plateau dose of three Wets daily, whereas the remaining nine subjects took wotablets as maximal dose. In the clomipramine group, 12patients the plateau dose was $50 \mathrm{mg}$ /day whereas tree patients did not take more than $25 \mathrm{mg} /$ day. The Finence between the two groups in number of tablets then daily was statistically significant $(p=.0007)$. Within each treatment group, a similar plateau dose was med in each treatment cycle: clomipramine $45.7 \pm$ $19 \mathrm{mg}$ (cycle 1), $48.3 \pm 3.8 \mathrm{mg}$ (cycle 2), and $50.0 \pm$ $13 \mathrm{mg}$ (cycle 3); placebo $2.6 \pm 0.1$ table (cycle 1), $26 \pm 0.1$ table (cycle 2), and $2.5 \pm 0.1$ tabl (cycle 3 ). The number of days on medication did not differ igrificantly between groups: clomipramine $12.6 \pm 0.9$ wocle 1), $12.1 \pm 0.8$ (cycle 2), and $13.1 \pm 0.9$ (cycle 3); pacebo $13.0 \pm 0.6$ (cycle 1), $14.0 \pm 0.7$ (cycle 2), and R2 \pm 0.8 (cycle 3 ).

\section{Sorm Concentrations of Clomipramine ond Desmethylclomipramine}

fortechnical reasons, serum concentrations were missmin eight subjects (four in the clomipramine group ad four in the placebo group). As expected given the bu dose and short duration of treatment, in the clomiprmine treated patients serum levels of clomipramine ad desmethylclomipramine were low (clomipramine enges: 30 to $190 \mathrm{nM}$, clomipramine mean \pm SEM: $90 \pm$
$13 \mathrm{nM}$; desmethylclomipramine ranges: 35 to $360 \mathrm{nM}$; desmethylclomipramine mean \pm SEM $126 \pm 27 \mathrm{nM}$ ). No detectable levels of clomipramine or desmethylclomipramine were found in the serum of placebotreated patients.

\section{Irritability, Depressed Mood, Tension, and Increased Appetite/Carbohydrate Craving}

The pre- and postmenstrual symptom rating (mean of 5 days; see Methods) of premenstrual irritability, depressed mood, tension, and increased appetite and/or carbohydrate craving during the two pretreatment reference cycles, the three treatment cycles, and the posttreatment reference cycle, are shown in Figures 1 to 4 . In addition, to illustrate the temporal relation between start of medication and onset of drug action, the daily irritability and depressed mood ratings throughout the study (group means) are shown in Figure 5 .

In both treatment groups, the mean ratings of premenstrual irritability, depressed mood, tension and increased appetite/carbohydrate craving during the three treatment cycles were significantly lower than the mean ratings of the two pretreatment reference cycles (see Figures 1 to 4 ) (clomipramine: irritability $p=.0007$, depressed $\operatorname{mood} p=.0008$, tension $p=.002$, increased appetite/carbohydrate craving $p=.01$; placebo: irrita-

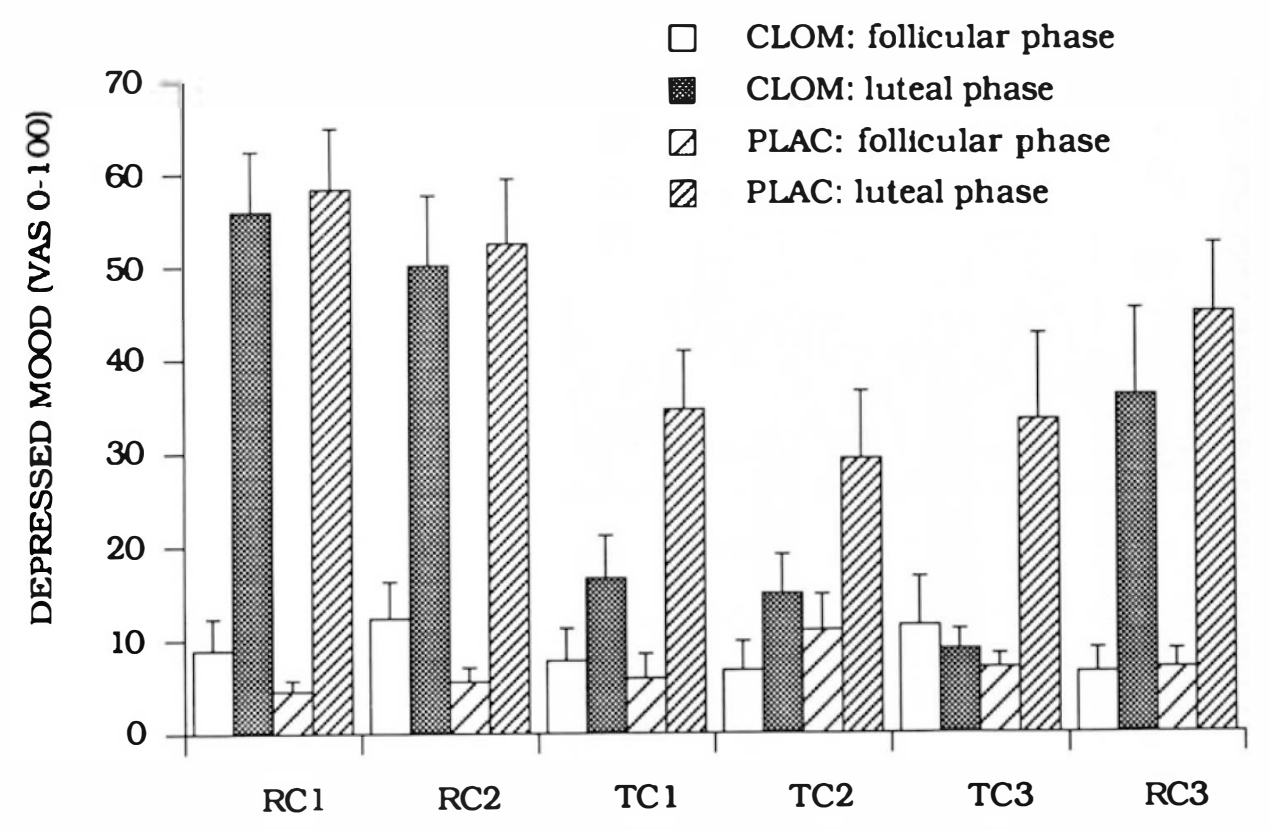

Fue 2. Means of self-rated depressed mood (VAS 0 to $100 \mathrm{~mm}$ ) of cycle day 6 to 10 (follicular phase) and of the 5 days preding the day of the menstruation (luteal phase). The bars represent group means with standard errors. During the woconsecutive pretreatment reference cycles (RC1 and $R C 2$ ) and during the posttreatment referencecycle (RC3), no treat5 in was given, whereas in the treatment cycles (TC1 to TC3) the patients were medicated with clomipramine or placebo penenstrually. Levels of significance (clomipramine group versus placebo group) for the premenstrual phases of each cycle ure: RC1 NS, RC2 NS, TC1 $p=.02$, TC2 NS ( $p=.07)$, TC3 $p=.03$, RC3 NS. For levels of significance when comparing teratings of the reference cycles with the rating of the treatment cycles within each treatment group, see Results. 


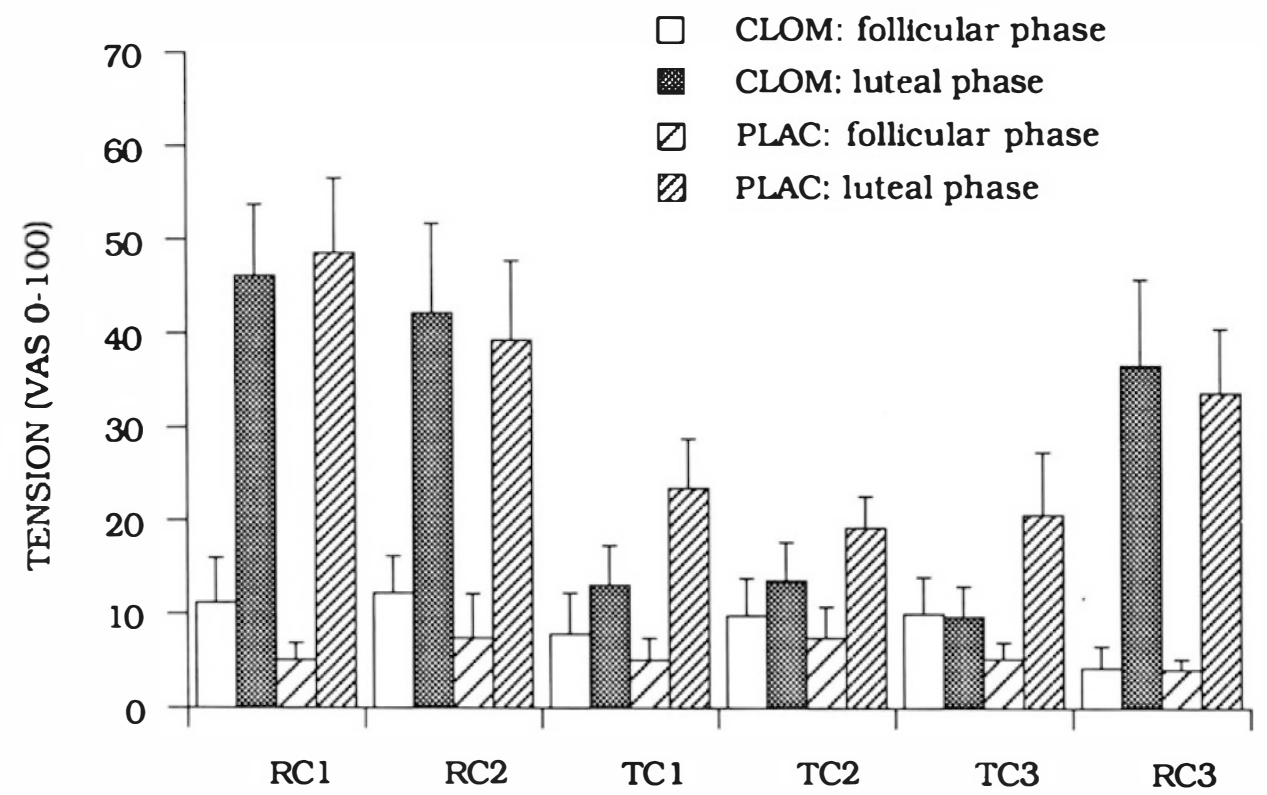

Figure 3. Means of self-rated tension (VAS 0 to $100 \mathrm{~mm}$ ) of cycle day 6 to 10 (follicular phase) and of the five days preceding the day of the menstruation (luteal phase). The bars represent group means with standard errors. During the two consect tive pretreatment reference cycles (RC1 and $R C 2)$ and during the posttreatment reference cycle (RC3), no treatment was given, whereas in the treatment cycles (TC1 to TC3) the patients were medicated with clomipramine or placebo premenstrually. Levels of significance (clomipramine group versus placebo group) for the premenstrual phases of each cycle were: RC1NS, RC2 NS, TC1 NS ( $p=.07)$, TC2 NS $(p=.1)$, TC3 $p=.05$, RC3 NS. For levels of significance when comparing the ratings of the reference cycles with the rating of the treatment cycles within each treatment group, see Results.

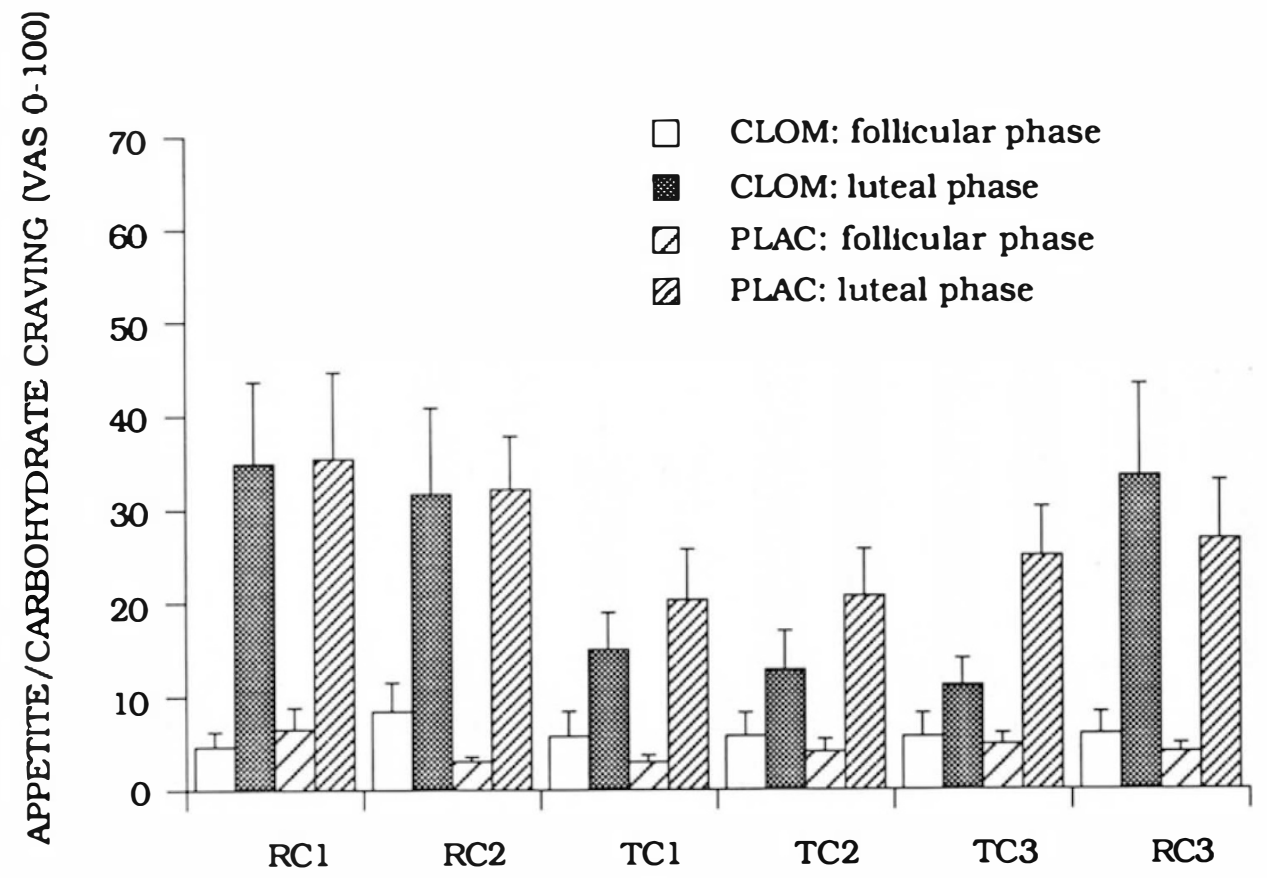

Figure 4. Means of self-rated increase in appetite/carbohydrate craving (VAS 0 to $100 \mathrm{~mm}$ ) of cycle day 6 to 10 (follicul phase) and of the 5 days preceding the day of the menstruation (luteal phase). The bars represent group means with star dard errors. During the two consecutive pretreatment reference cycles (RC1 and RC2) and during the posttreatment refer. ence cycle (RC3), no treatment was given, whereas in the treatment cycles (TC1 to TC3) the patients were medicated with clomipramine or placebo premenstrually. Differences between the two treatment groups never reached statistical significant TC1 $p=.3$, TC2 $p=.1$, TC3 $p=.07$. For levels of significance when comparing the ratings of the reference cycles with the rating of the treatment cycles within each treatment group, see Results. 

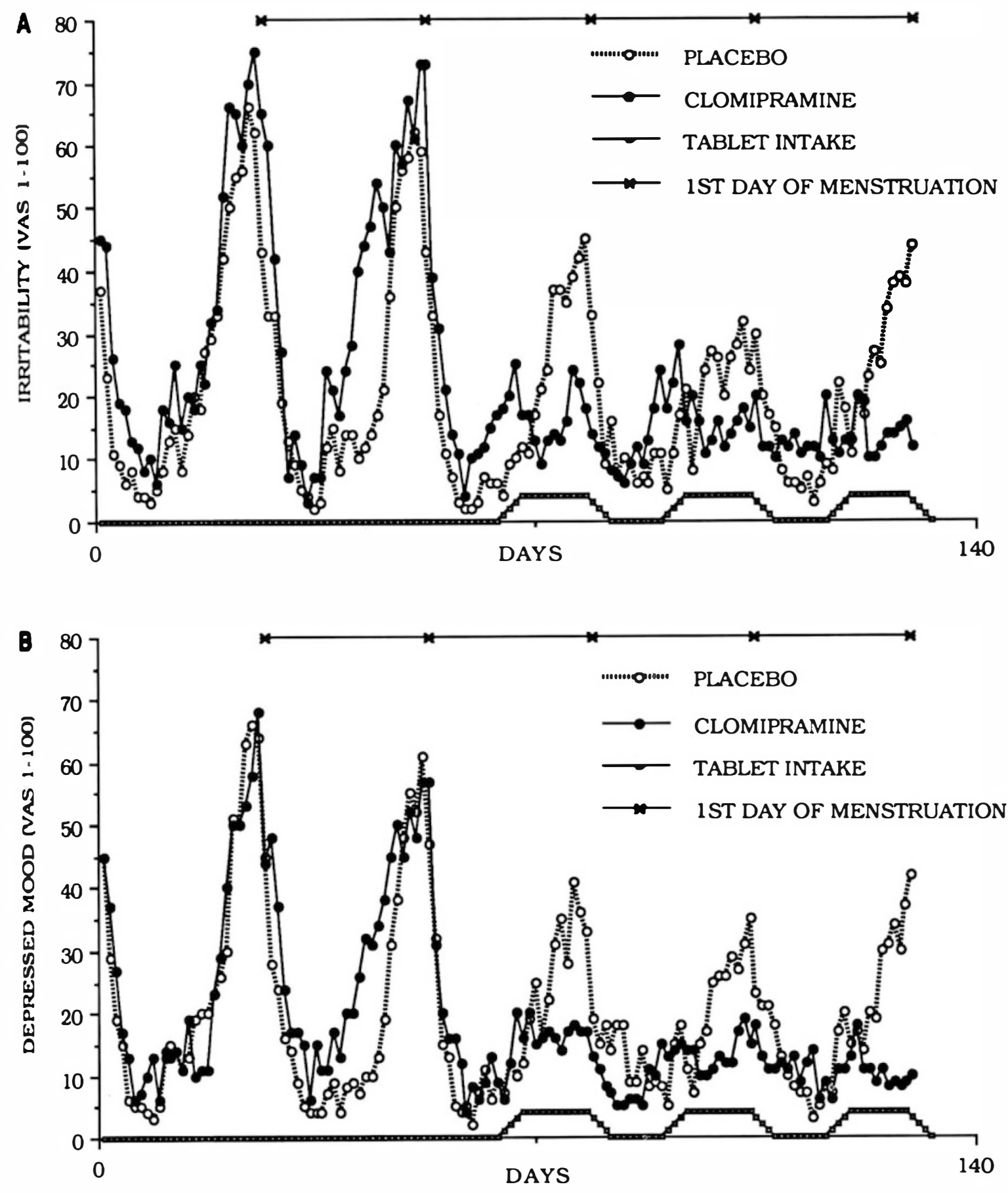

Fore 5. Daily mean rating of irritability (A) and depressed mood (B) during two reference cycles and three treatment gdes in subjects treated with clomipramine and placebo, respectively. SEM are omitted for clarity. Also indicated are first dy of menstruation (top) and the scheme for dose escalation (0/10/25/50 $\pm 25 \mathrm{mg} /$ day) (bottom).

Lity $p=.001$, depressed $\operatorname{mood} p=.002$, tension $p=$ $\mathbb{m}$, increased appetite/carbohydrate craving $p=01$ ). Wether in the clomipramine group nor in the placebo poup did the rating of the third treatment cycle differ ignificantly from the rating of the first treatment cycle vithrespect toany of these four symptoms. During the posttreatment reference cycle, a reappearance of symp- toms was observed in both groups. Thus, in the clomipramine group the mean rating of the posttreatment reference cycle was significantly higher than that of the three treatment cycles with respect to irritability $(p=$ $.003)$, depressed mood $(p=.008)$, tension $(p=.009)$, and increased appetite/carbohydrate craving $(p=.03)$. In the placebo group the mean rating of the posttreat- 
ment reference cycle was significantly higher than that of the three treatment cycles with respect to irritability $(p=.006)$, depressed $\operatorname{mood}(p=.04)$, and tension $(p=$ $.04)$, but not with respect to increased appetite/carbohydrate craving.

The mean premenstrual irritability, depressed mood, tension, and appetite/carbohydrate craving ratings during the two pretreatment cycles and during the posttreatment reference cycle were not significantly different in the clomipramine group as compared to the placebo group. In contrast, the mean ratings of premenstrual irritability, depressed mood, and tension of the three treatment cycles were all significantly lower in the clomipramine group than in the placebo group (irritability: $p=.02$; depressed mood: $p=.01$; tension: $p=$ .04). With respect to increased appetite and/or carbohydrate craving (mean of all three treatment cycles), the difference between the clomipramine group and the placebo group did not reach statistical significance when all subjects were included in the calculation $(p=.07)$. However, since premenstrual changes in appetite and/ or carbohydrate craving was not an inclusion criterion, some participants did not display a premenstrual increase in appetite or carbohydrate craving even during the pretreatment reference cycles. When only patients with a mean pretreatment premenstrual rating of increased appetite/carbohydrate craving exceeding $20 \mathrm{~mm}$ (9 women in each group) were included in the analy- sis, the mean premenstrual rating of these symptoms during the three treatment cycles, but not the mean rating during the pretreatment reference cycles or during the posttreatment reference cycle, was significantly lower in the clomipramine group than in the placebo group $(p=.04)$.

\section{Breast Tenderness and Bloating}

The mean premenstrual ratings of the somatic symptoms breast tenderness and bloating during treatment cycles 3 to 5 were significantly lower than the corre sponding rating during the pretreatment reference cycles in the clomipramine group (both symptoms: $p=$ .003 ) but not in the placebo group (Figs. 6 and 7). How. ever, when interpreting this difference in symptom de crease between the two groups, it should be noted that both these symptoms accidentally were more common in the clomipramine group than in the placebo group during the pretreatment reference cycles (although this difference did not reach statistical significance). When the clomipramine and placebo groups were compared with respect to mean premenstrual ratings of breast tenderness and bloating during the three treatmentcycles, no statistically significant differences were observed. Also in the posttreatment reference cycle, the two groups did not differ significantly with respect to breast tenderness or bloating.

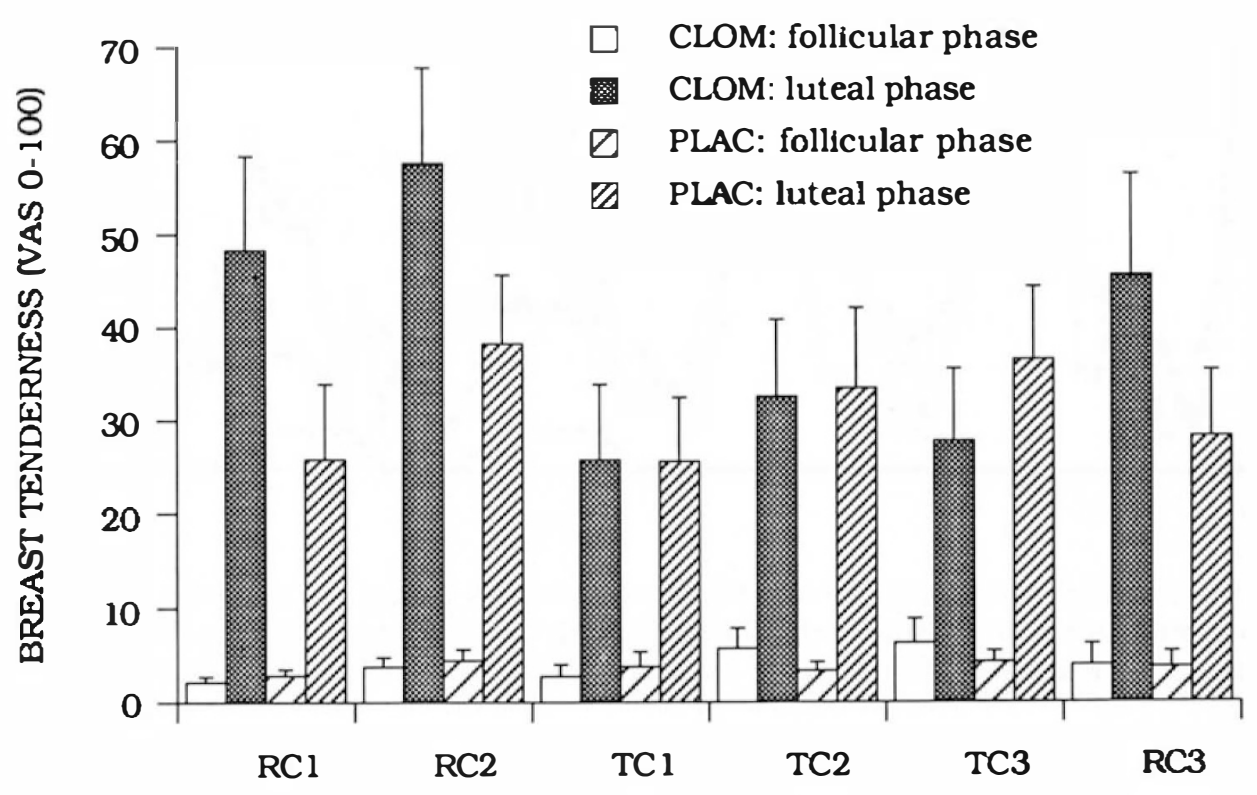

Figure 6. Means of self-rated breast tenderness (VAS 0 to $100 \mathrm{~mm}$ ) of cycle day 6 to 10 (follicular phase) and of the 5 drp preceding the day of the menstruation (luteal phase). The bars represent group means with standard errors. During the two consecutive pretreatment reference cycles (RC1 and RC2) and during the posttreatment reference cycle (RC3), no tres ment was given, whereas in the treatment cycles (TC1 to TC3) the patients were medicated with clomipramine or placet premenstrually. Differences between the two treatment groups never reached statistical significance. For levels of significan when comparing the ratings of the reference cycles with the rating of the treatment cycles within each treatment group see Results. 


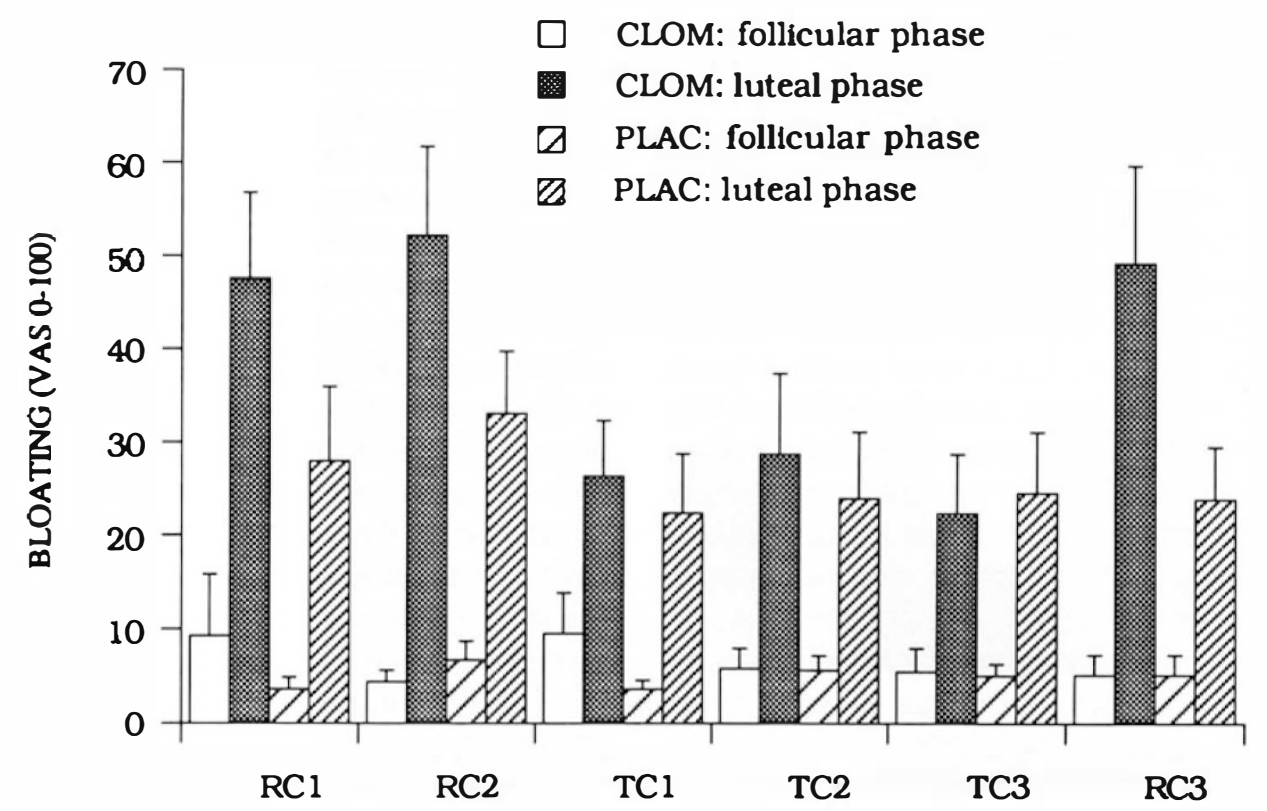

Fure 7. Means of self-rated bloating (VAS 0 to $100 \mathrm{~mm}$ ) of cycle day 6 to 10 (follicular phase) and of the 5 days preceding the day of the menstruation (luteal phase). The bars represent group means with standard errors. During the two consecuine pretreatment reference cycles (RC1 and RC2) and during the posttreatment reference cycle (RC3), no treatment was men, whereas in the treatment cycles (TC1 to TC3) the patients were medicated with clomipramine or placebo premenstrually. atrences between the two treatment groups never reached statistical significance. For levels of significance when comparif the ratings of the reference cycles with the rating of the treatment cycles with each treatment group, see Results.

\section{Souse's Rating}

blependent rating of the irritability and depressed mod displayed by the patient in the premenstrual phese was undertaken in at least one of the pretreatment reference cycles and in at least one of the treatment cycles by the spouses of 17 of the patients (11 in clomipramine group, six in the placebo group). Mrereas spouse's rating did not differ significantly be- tween groups during the two pretreatment reference cycles, the rating of irritability and depressed mood during the treatment cycles was significantly lower in the clomipramine treated group (see Fig. 8).

\section{Global Assessment}

As shown in Figure 9, self-rated global improvement during treatment was significantly more favorable

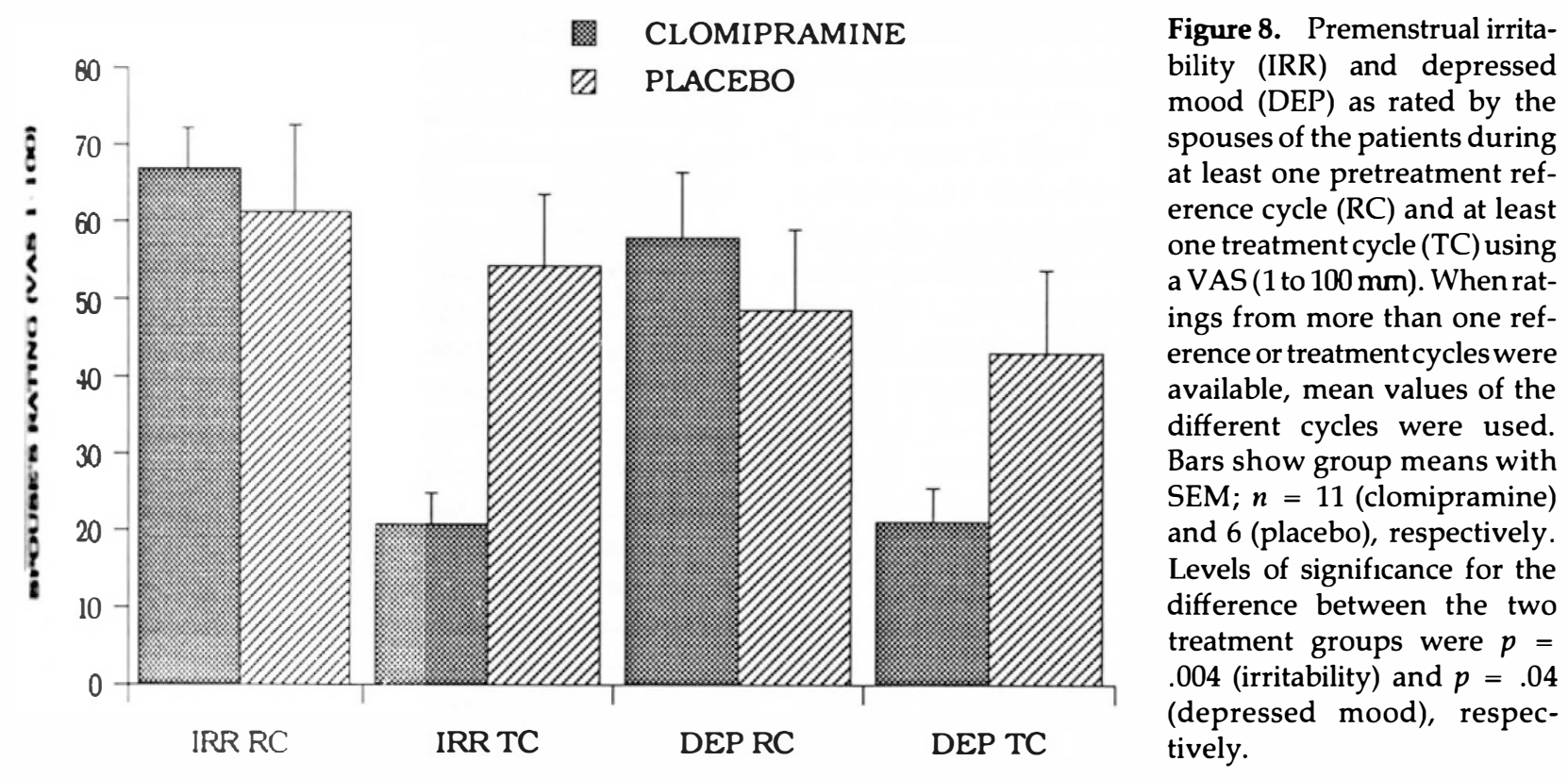




\section{GLOBAL ASSESSMENT}

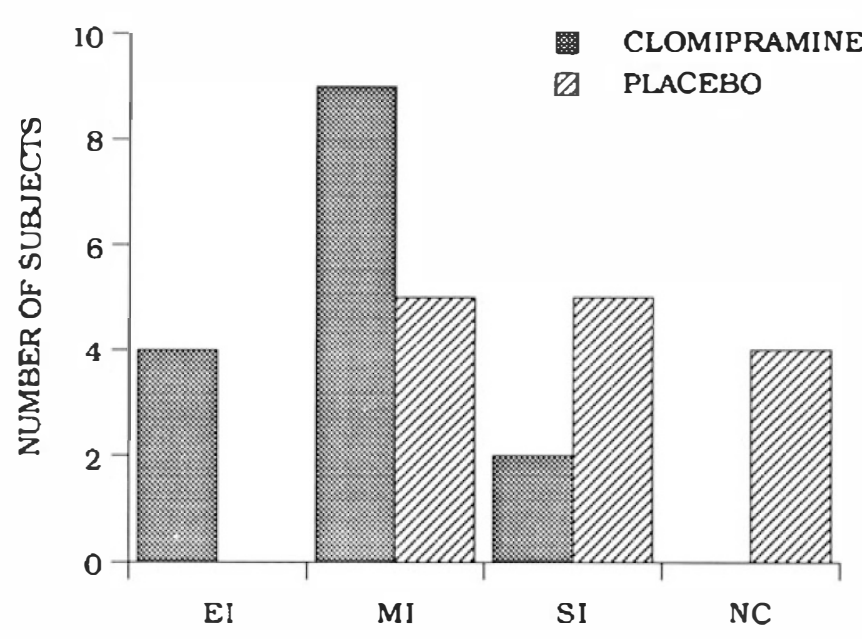

Figure 9. Global improvement during treatment as assessed shortly after the last treatment cycle by the subjects completing the trial. Bars represent number of subjects. No participants reported a deterioration of premenstrual complaints during medication. $\mathrm{EI}=$ enormously improved, $\mathrm{MI}=$ much improved, SI = somewhat improved, NC = no change. The difference between the two groups was statistically significant $(p=.002)$.

in the clomipramine group than in the group given placebo.

\section{Tolerability Data}

All symptoms reported as possible side effects of the treatment by the patients completing the trial are shown in Table 1. In addition, six subjects in the clomipramine group withdrew from treatment due to one or several unwanted effects. Five of these reported increased fatigue, four reported dry mouth, one reported increased anxiety and suicidal thoughts, one reported sweating, and one reported vertigo.

Table 1. Side Effects of Subjects Completing the Trial

\begin{tabular}{lcl}
\hline \multicolumn{1}{c}{ Symptom } & $\begin{array}{c}\text { Clomipramine } \\
(\boldsymbol{n}=\mathbf{1 5})\end{array}$ & $\begin{array}{c}\text { Placebo } \\
(\boldsymbol{n}=\mathbf{1 4})\end{array}$ \\
\hline Dry mouth & $10(67 \%)$ & $1(7 \%)$ \\
Fatigue & $8(53 \%)$ & $2(14 \%)$ \\
Vertigo & $5(33 \%)$ & 0 \\
Nausea & $4(27 \%)$ & $2(14 \%)$ \\
Sweating & $2(13 \%)$ & $1(7 \%)$ \\
Headache & $2(13 \%)$ & $1(7 \%)$ \\
Obstipation & $2(13 \%)$ & 0 \\
Disturbance of sleep & $1(7 \%)$ & $1(7 \%)$ \\
Sexual side effects & $1(7 \%)$ & 0 \\
Formication & $1(7 \%)$ & 0 \\
Increased thirst & 0 & $1(7 \%)$ \\
\hline
\end{tabular}

\section{DISCUSSION}

The present trial confirms our previous observation (Eriksson et al. 1990; Sundblad et al. 1992) that low doses of the tricyclic antidepressant clomipramine is more effective than placebo in reducing premenstrual irritability and depressed mood. Thus, both with re spect to the daily rating of these symptoms and with respect to the global assessment of treatment, the women in the clomipramine group reported significantly greater improvement than did patients treated with placebo. The superiority of clomipramine to placebo also lends support from the symptom rating performed bythe patients' spouses; however, due to a large number of missing data, the outcome of the spouses' rating shouldbe interpreted with some caution.

Clomipramine is a strong although nonselective serotonin reuptake inhibitor (Carlsson et al. 1969; Hyt. tel and Larsen 1985). Given the postulated involvement of serotonin in the control of mood and irritability as well as in other symptoms that may be part of PMS, such as anxiety and increased carbohydrate craving (see Eriksson and Humble 1990), it is tempting to suggest that the drug reduces premenstrual complaints by facilitating serotonergic neurotransmission. In support for this assumption, recent reports indicate that the selective serotonin reuptake inhibitor fluoxetine, ad. ministered continuously throughout the menstrual cy. cle, may also be effective in the treatment of PMS (Rickels et al. 1990; Stone et al. 1991; Menkes et al. 1992; Wood et al. 1992). Moreover, the serotonin selectivere uptake inhibitor paroxetine appears superior to the noradrenaline selective reuptake inhibitor maprotiline in the treatment of severe premenstrual complaints $(\mathrm{M}$. Hedberg, C. Sundblad, B. Andersch, and E. Eriksson to be published). The apparent lack of effect of theserotonin reuptake inhibitor fluvoxamine on premenstrual complaints in the study by Veeninga et al. (1990) may be due to shortcomings in the trial design; for example, in this trial the diagnosis of premenstrual syndrome was not confirmed by daily symptom ratings beforethe initiation of treatment.

Women with severe premenstrual irritability and/or depression (and fulfilling the diagnostic criteria of LLPDD according to DSM-III-R) were included in the present study regardless of whether they displayed also other premenstrual complaints or not. Consequently, the effect evaluation was focused on irritability and depressed mood (which we believe are the core symp. toms of PMS). However, in addition, the occurrence and severity of tension, increased appetite and/or car. bohydrate craving, breast tenderness, and bloating were also rated daily by the participants. Whereas ten sion was significantly more reduced by clomipramine than by placebo, the effects of clomipramine on the somatic symptoms premenstrual breast tenderness and 
bloating were less impressive. The finding that mental symptoms, such as irritability and depression, respond markedly to a treatment not affecting the somatic sympbons of PMS may lead to the questioning of whether these two groups of symptoms are indeed parts of the ame syndrome; alternatively, they should be regarded sepiphenomena, both related to the cyclical variation n ovarial activity but unrelated to each other and ocouring irrespectively of each other. Hence, assessment d the severity of PMS by calculating the sum of the rating scores of a series of somatic and mental symptoms nay be inappropriate.

According to the assumed involvement of serotoin in the regulation of food intake (Wurtman 1990), a serotonin reuptake inhibitor should be expected to reduce appetite and carbohydrate craving. However, wight gain rather than weight loss is a common side fect of clomipramine when used for, for example, depression. Because the effect of more selective seromin reuptake inhibitors is generally a decrease in wight (Wise 1992), this effect of clomipramine is probthy not due to the effect of the drug on serotonin reptake; according to one hypothesis, the paradoxical fiect of clomipramine on weight is due to serotonin-2 mextor antagonism (Bernstein 1987). In line with previos studies on premenstrual changes in food intake Both-Ortman et al. 1988), many, but not all, of the pariopants in the present trial displayed increased appeacarbohydrate craving premenstrually. Interestingly, donipramine, more effectively than placebo, reduced tis symptom; moreover, no patients reported weight in as a side effect of active treatment.

When used for the treatment of depression, panic sorder and OCD, clomipramine, as well as other moamine reuptake inhibitors, usually requires bemeen 1 and 2 weeks of treatment before marked beficial effects are evident and often several months Wore a maximal therapeutic response is achieved (Katz d. 1990; Modigh et al. 1992). For this reason, it has ben questioned whether the serotonin (and/or nortenaline) reuptake inhibiting effects, which occur torty after drug intake, indeed constitute the primary ode of action of the drugs. The most important condrsion of the present study is that a marked beneficial fot of clomipramine on depressed mood and irritain the premenstrual phase does not require conmous medication (Sundblad et al. 1992) but can be dieved by intermittent treatment starting at ovulaand terminating a few days after the onset of the monstrual bleeding. Hence, the time lag between start Inedication and clinical effect is considerably shorter then clomipramine is used for PMS than when the tog is used for depression, panic disorder, or OCD. deed, only 7 days after treatment was initiated, and by 4 days after the maximal dose of 50 to $75 \mathrm{mg}$ had ten reached, a marked symptom reduction was ob- served in patients receiving active treatment (see Fig. 5 ). Whether the effect lag when using clomipramine for PMS is even shorter than four to seven days is difficult to decide from the present data. Notably, many PMS patients that we have treated with clomipramine in the premenstrual phase claim that a beneficial effect may be experienced within less than 24 hours from drug intake provided that a dose of at least $25 \mathrm{mg}$ is taken as starting dose; however, to exclude the placebo factor this impression has to be confirmed in a placebo-controlled trial. Interestingly, according to a previous report by Brezinski and coworkers (1990), the serotoninreleasing agent fenfluramine also reduces premenstrual depression and carbohydrate craving when administered intermittently during luteal phases only. Also, a recent case report supports that fluoxetine has a very short onset of action when used for the treatment of premenstrual complaints (Daamen and Brown 1992).

The difference in time lag when using clomipramine for PMS on the one hand, and for depression, panic disorder, and OCD on the other, may lead to the suggestion that different modes of actions are involved when using the drug for the different conditions. Alternatively, and perhaps more likely, the serotonin reuptake inhibition may always be the primary mode of action for clomipramine; however, some conditions may require a longer period of increased serotonergic activity before symptoms are remitted than others. The possibility that certain symptoms are likely to respond more quickly to antidepressants than others, regardless of the underlying condition, should also be considered. Irritability is one of the symptoms of PMS that responds quickly and dramatically to treatment with clomipramine; in depressed patients, hostility has recently been shown to respond more rapidly to antidepressants than do other features of the depressive syndrome (Katz et al. 1991).

The dosage required when using clomipramine (and probably also other serotonin reuptake inhibitors) also differs markedly depending on the condition that is to be treated. Thus, whereas $150 \mathrm{mg}$ is usually recommended as a daily dosage for the treatment of depression, even higher doses are often required for the treatment of OCD. In contrast, both panic disorder (Gloger et al. 1981; Modigh et al. 1989) and PMS (Eriksson et al. 1990; Sundblad et al. 1992; present trial) usually respond to considerably lower doses (i.e., 25 to $75 \mathrm{mg}$ per day). As would be expected from the drug regimen applied, plasma concentrations of clomipramine and its metabolite desmethylclomipramine were low as compared to the concentrations normally recommended for the treatment of depression.

Because no group given continuous medication with clomipramine was included in the present trial, it is not possible to conclude whether clomipramine given intermittently in the luteal phase only is as effec- 
tive as clomipramine given every day of the cycle. A comparison of the outcome of this trial and that of our earlier study on clomipramine given throughout the cycle (Sundblad et al. 1992) indicates that continuous medication is in fact somewhat more effective than is intermittent treatment. Thus, the symptom reduction in percent of the ratings during the reference cycles was slightly higher in the trial using continuous medication ( $80 \%$ versus $70 \%$ ). When comparing the two different trials, however, it should be noted that the patients reached somewhat higher mean doses of clomipramine in the trial using continuous medication.

In our experience, women with PMS, like patients with panic disorder (Modigh et al. 1992), are more sensitive to the side effects of clomipramine, such as sweating, dry mouth, and sexual impairment, than are depressed patients. For this reason, the possibility that drug treatment can be restricted to the luteal phase and still be effective against PMS is of obvious practical importance, especially for women with premenstrual complaints lasting for less than 1 week before the menstruation. On the other hand, for patients with symptoms of PMS starting around ovulation and terminating a few days after the menstrual bleeding has started, the symptom-free interval may be so short that a tapering of the medication is not meaningful. Also, it should be pointed out that for some patients, continuous administration of clomipramine over the entire menstrual cycle may be associated with milder side effects than intermittent administration. Thus, sometimes the most cumbersome unwanted effects of clomipramine are those appearing during the first days of treatment only to fade and vanish after a few days or weeks of drug administration. When using intermittent administration of clomipramine, in some patients this syndrome may reappear every time the patient starts the medication, that is, once every menstrual cycle; in contrast, when clomipramine treatment is chronic these symptoms are usually a problem only during the first treatment cycle. Notably, the number of drop outs due to side effects was larger in the present trial than in our previous study of clomipramine in PMS (Sundblad et al. 1992), probably due to the fact that the escalation of dose was faster and that initial side effects in some patients reappeared for every new treatment period. Needless to say, like in most drug trials using tricyclic antidepressant drugs, it cannot be excluded that the occurrence of side effects to some extent may have reduced the blindness on the part of the participants.

A third treatment strategy that we have successfully applied openly in a large number of PMS patients is the following. A very low dose of clomipramine (5 to $10 \mathrm{mg}$ ) is taken daily throughout the menstrual cycle; in the beginning of the luteal phase, the patient increases the dosage to a maximal dose of 25 to $75 \mathrm{mg}$, and when the menstrualbleeding starts the medication is again tapered to the low maintenance dose. With this regimen, the reappearance of initial side effects is avoided, other side effects are minimized (especially during the follicular phase), and an impressive efficacy is achieved (C. Sundblad and E. Eriksson, unpublished observation). Also, the possibility that an intermittent administration is rendered more effective if preceded by a few cycles of continuous medication should be taken into consideration (C. Sundblad and E. Eriksson, unpublished observation).

In conclusion, the present trial demonstrates a shot onset of action when using clomipramine for the treatment of PMS. We suggest that when clomipramine is used for this indication, the drug regimen should be individualized; thus, for some subjects continuous medication is preferable, whereas for others intermittent treatment appears much more acceptable.

\section{ACKNOWLEDGMENTS}

This study was supported by grants from the Swedish Medical Research Council (04752 and 08668), the Swedish Society of Medicine, and by CIBA-GEIGY Pharma Division, Sweden. Margareta Lundgren and Norunn Persson are gratefully $x$. knowledged for excellent technical assistance.

\section{REFERENCES}

American Psychiatric Association (1985): Diagnostic and Statistical Manual of Mental Disorders, 3rd ed, revised version. Washington DC, APA Press, pp 367-369

Andersch B, Wendestam C, Hahn L, Öhman R (1986): Premenstrual complaints. I. Prevalence of premenstrual symptoms in a Swedish urban population. J Psychosom Obstet Gynaecol 5:39-49

Bernstein JG (1987): Induction of obesity of psychotropix drugs. Ann NY Acad Sci 499:203-215

Both-Ortman B, Rubinow DR, Hoban MC, Malley J, Grove GN (1988): Menstrual cycle phase-related change in appe tite in patients with premenstrual syndrome and in con trol subjects. Am J Psychiatry 145:628-631

Breziniski AA, Wurtman JJ, Wurtman RJ, Gleason R, Green field J, Nader T (1990): d-Fenfluramine suppresses the increased calorie and carbohydrate intake and improves the mood of women with premenstrual depression. $0 \mathrm{~b}$ stet Gynecol 76:296-301

Carlsson A, Corrodi H, Fuxe K, Hökfelt T (1969): Effectd antidepressant drugs on the depletion of intraneurond brain 5-hydroxytryptamine stores caused by 4-methy$\alpha$-ethyl-meta-tyramine. Eur J Pharmacol 5:357-366

Chihal HJ (1987): Indications for drug therapy in premenstnu syndrome patients. J Reprod Med 32:449-452

Clomipramine Collaborative Study Group (1991): Clomipr mine in the treatment of patients with obsessive-com pulsive disorder. Arch Gen Psychiatry 48:730-738

Daamen MJ, Brown WA (1992): Single-dose fluoxetine in management of premenstrual syndrome. J Clin $\mathrm{P}_{5}$ chiatry 53:210-211 
Eriksson E, Humble M (1990): Serotonin in psychiatric pathophysiology. A review of data from experimental and clinical research. In Pohl R, Gershon S (eds), The Biological Basis of Psychiatric Treatment. Progress in Basic Clinical Pharmacology. Basel, Karger, pp 66-119

Enksson E, Lisjö P, Sundblad C, Andersson K, Andersch B, Modigh K (1990): Clomipramine in the premenstrual syndrome. Acta Psychiatr Scand 81:87-88

fank RT (1931): The hormonal causes of premenstrual tension. Arch Neurol Psychiatry 26:1053-1057

Coger S, Grunhaus L, Birmacher B, Troudart T (1981): Treatment of spontaneous panic attacks with clomipramine. Am J Psychiatry 138:1215-1217

Hroreich U, Endicott J, Schnacht S, Nee J (1982): The diversity of premenstrual changes as reflected in the Premenstrual Assessment Form. Acta Psychiat Scand 65:46-65

Hyttel J, Larsen J-J (1985): Neuropharmacological mechanisms of serotonin uptake inhibitors with antidepressant activity. In Naranjo CA, Sellers EM (eds), Recent Advances in New Pyschopharmacological Treatments for Alcoholism. Amsterdam, Elsevier, pp 107-119

K女 RJ, DeVaugh-Geiss J, Landau P (1990): Clomipramine in obsessive-compulsive disorder. Biol Psychiatry 28: 401-414

toMM, Koslow SH, Maas JW, Frazer A, Kocis J, Secunda S, Bowden CL, Casper RC (1991): Identifying the specific dinical actions of amitriptyline: Interrelationships of behaviour, affect and plasma levels in depression. Psychol Med 21:599-611

Merkes DB, Taghavi E, Mason PA, Spears GFS, Howard RC (1992): Fluoxetine treatment of severe premenstrual syndrome. Br Med J 305:346-347

Udigh K, Eriksson E, Lisjö P, Westberg P (1989): Follow-up study of clomipramine in the treatment of panic disor- der. In Achté K, Tamminen T, Laaksonen R (eds), Many Faces of Panic Disorder. Proceedings of the WPA Symposium on the Psychopathology of Panic Disorders. Psychiatrica Fennica Supplementum. Hanko, Finland, Foundation of Psychiatric Research in Finland, pp 145-153

Modigh K, Westberg P, Eriksson E (1992): Superiority of clomipramine over imipramine in the treatment of panic disorder: A placebo-controlled trial. J Clin Psychopharmacol 12:251-261

Nyberg G, Martensson E (1984): Determination of free fractions of tricyclic antidepressants. Naunyn Schmiedebergs Arch Pharmacol 327:260-265

Reid RL (1985): Premenstrual syndrome. Curr Probl Obstet Gynecol Fertil 8:7-9

Rickels K, Freedman EW, Sondheimer S, Albert J (1990): Fluoxetine in the treatment of premenstrual syndrome. Curr Ther Res 48:161-166

Sundblad C, Andersch B, Modigh K, Eriksson E (1992): Clomipramine effectively reduces premenstrual irritability and dysphoria: A placebo-controlled trial. Acta Psychiatr Scand 85:39-47

Veeninga AT, Westenberg HGM, Weusten JTN (1990): Fluvoxamine in the treatment of menstrually related mood disorders. Psychopharmacology 102:414-416

Wise SD (1992): Clinical studies with fluoxetine in obesity. Am J Clin Nutr 55 (Suppl 1):181S-184S

Wood SH, Mortola JF, Chan YF, Moossazadeh F, Yen SS (1992): Treatment of premenstrual syndrome with fluoxetine: A double-blind, placebo-controlled, crossover study. Obstet Gynecol 80:339-344

Wurtman JJ (1990): Carbohydrate craving. Relationship between carbohydrate intake and disorders of mood. Drugs 39 (Suppl 3):49-52 